\title{
Few-cycle self-compression via multimode nonlinear optics in gas filled waveguides
}

\author{
P. N. Anderson ${ }^{1}$, T. J. Butcher ${ }^{1}$, P. Horak ${ }^{1}$, J. G. Frey ${ }^{2}$, W. S. Brocklesby ${ }^{1}$ \\ 1. Optoelectronics Research Centre, University of Southampton, UK \\ 2. School of Chemistry, University of Southampton, UK \\ Author e-mail address: pna1g10@orc.soton.ac.uk
}

\begin{abstract}
Multimode simulations predict dramatic ionization-induced self-compression of high energy ultrashort pulses within short gas filled capillaries. The mechanism observed allows for the temporal compression of $53 \mathrm{fs}$ pulses into the few-cycle regime.

OCIS codes: (320.5520) Pulse compression; (020.2649) Strong field laser physics
\end{abstract}

\section{Introduction}

Demands in tabletop high field physics applications such as high harmonic generation (HHG) and laser wakefield acceleration drive the development of high energy laser sources whose pulses are temporally compressed to ever shorter durations. In this high intensity regime, state of the art pulse compressors rely on hollow capillary compression or filamentation to achieve the required degree of temporal and spectral reshaping. Both of these techniques require very long interaction lengths and complex optical components are often necessary to counteract for dispersive effects.

In 2004 Wagner et al discovered an alternative self-compression technique to filamentation [1], which led to the compression of a $28.9 \mathrm{fs}$ pulse to $13.3 \mathrm{fs}$ within a $25 \mathrm{~mm}$ long capillary filled with $5 \mathrm{mbar}$ of argon. This geometry is of particular interest as the temporal compression and spatial confinement of the pulse within the capillary, coupled with the relatively low gas pressures required make it ideal for performing pulse compression and high field physics experiments in-situ.

In this work we greatly enhance the degree of temporal compression achievable via this technique by extending it into the highly nonlinear regime encountered when significant densities of plasma are generated. We perform complex numerical simulations to analyze the full multimode propagation dynamics of a 53 fs FWHM transform limited pulse as it propagates through a short length of capillary filled with $150 \mathrm{mbar}$ of argon. We have observed how the combined effects of intermodal walk-off, ionization driven nonlinear mode-mixing and spectral broadening can lead to the formation of a spatially compressed pulse with a FWHM as short as $8 \mathrm{fs}$. The mechanism developed is scalable to various input pulse energies and durations via the selection of appropriate capillary dimensions, gas densities and gas types. Preliminary experimental work suggests a strong agreement with our theoretical results.

\section{Model and results}

The model described here is based on a multimode-generalized nonlinear Schrödinger equation (MM-GNLSE) [2, 3]. This advanced three dimensional model simulates a vast array of effects including dispersion, neutral gas nonlinearity, plasma nonlinearity, ionization induced losses and self-steepening.

The result of one of these simulations is shown in figure 1. The central wavelength of the input pulses was $790 \mathrm{~nm}$, the pulse energy was $805 \mu \mathrm{J}$ and the FWHM was $53 \mathrm{fs}$. The capillary design used here [3] was $70 \mathrm{~mm}$ in length with a $150 \mu \mathrm{m}$ core diameter and gas inlets positioned $20 \mathrm{~mm}$ from each end respectively. Argon was selected for this work and its peak pressure within the capillary was fixed at $150 \mathrm{mbar}$. The near Gaussian input pulse undergoes extreme spatio-temporal reshaping as it propagates though the length of capillary. This reshaping can be understood by analyzing the on-axis complex modal envelopes, figure 1(c) and (d). Although the majority of the input power is coupled into the fundamental $E H_{1,1}$ capillary mode, power is also coupled into higher order modes at the input to the capillary. Linear beating between these modes leads to regions of high intensity spaced along the length of the capillary. Within these regions the high fraction of plasma generated by the leading edge of the pulse rapidly couples light into the first higher-order mode, leading to a steepening of its trailing edge and considerable spectral broadening. This ionization-induced nonlinear mode-mixing process appears to cascade through successively higher order modes, producing the temporally compressed train of modal envelopes shown in figure 1(d) (note that only the three lowest-order modes are plotted for clarity). Upon the reconstruction of the full optical field at a given point in the capillary it is observed that the superposition of the temporally compressed higher-order modes leads to the formation of few-cycle pulses with FWHM as short as 8 fs. Further simulations 
have shown this technique of compression to be applicable to input pulses with various energies and durations if alternative capillary dimensions, different gas densities or a gas with a more suitable ionization potential is selected.
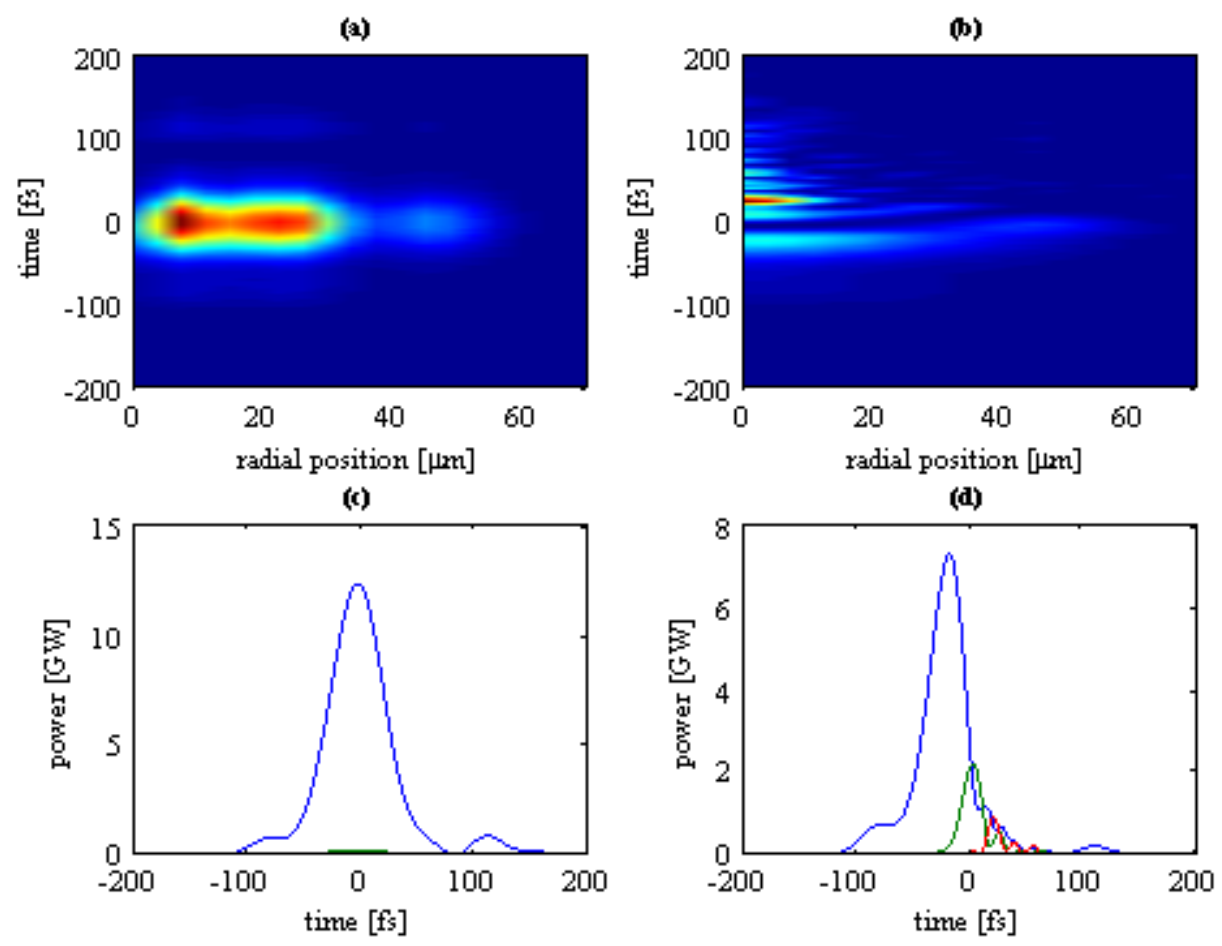

Fig. 1. (a) The spatio-temporal profile of the $805 \mu J 53$ fs pulse at the entrance to the capillary. (b) The spatially and temporally compressed pulse produced via propagation through a $70 \mathrm{~mm}$ length of capillary filled with $150 \mathrm{mbar}$ of argon. (c) The on-axis modal envelopes at the entrance to the capillary. (d) The same envelopes at the exit of the capillary. Nonlinear mode-mixing has coupled significant quantities of optical power into the first two higher-order modes (green and red curves); the superposition of which leads to the formation of pulses as short as $8 \mathrm{fs}$.

Experimentally we have discovered that optimizing this self-compression process to maintain a spatially compressed few-cycle pulse over several centimeters can lead to dramatic increases (two orders of magnitude) in the high harmonic flux generated within the capillary. We contribute this to the fact that the steep leading edge of the compressed pulse greatly enhances the phase matching of the HHG process. As this particular capillary was developed to also compress the few-cycle pulses spatially their divergence is very high upon exit from the capillary. This divergence can also be controlled by varying the capillary dimensions, making coupling few-cycle pulses out of a short gas filled capillary a promising possibility.

\section{Conclusions and outlook}

We have used an advanced multimode propagation model to predict the spatio-temporal dynamics of an ultrashort high energy pulse as it propagates through a short length of argon filled capillary. We have observed that the combined effects of several multimode phenomena can lead to the dramatic compression of a 53 fs transform limited pulse into the few cycle regime. We are currently working towards further verifying these theoretical predictions in the laboratory. We will achieve this by observing a predicted shift in the cut-off energy of the high harmonic radiation generated within the capillary.

\section{References}

[1] N. L. Wagner, E. A. Gibson, T. Popmintchev, I. P. Christov, M. M. Murnane and H. C. Kapteyn, "Self-Compression of Ultrashort Pulses through Ionization-Induced Spatiotemporal Reshaping", Phys. Rev. Lett. 93, 173902 (2004)

[2] F. Poletti and P. Horak, "Description of ultrashort pulse propagation in multimode optical fibers", J. Opt. Soc. Am. B 25, 1645(2008)

[3] R.T.Chapman, T.J.Butcher, P.Horak, F.Poletti, J.G.Frey, W.S.Brocklesby, "Modal effects on pump-pulse propagation in an Ar-filled capillary", Optics Express 18, 13279 (2010) 